\title{
Kommissionierung par excellence
}

\author{
DiPL.-Wi.-ING. MARTIN GLIESCHE, \\ DIPL.-KFF. JOHANNA HAAG \\ FACHGEBIET LOGISTIK, UNIVERSITÄT DORTMUND
}

\section{Zusammenfassung}

Der Kommissionierprozess stellt im Rahmen der innerbetrieblichen Logistik - gerade auch im Hinblick auf Just-In-Time-Lieferungen und Fragen der Produkthaftung - einen zentralen Baustein des Materialund Informationsflusses in Unternehmen dar. Dabei ist die Wahl des Kommissioniersystems ausschlaggebend für die Optimierung der personal- und zeitaufwendigen Kommissioniervorgänge und dient damit zur Leistungssteigerung unter gleichzeitiger Reduzierung der Fehlerquote.

\begin{abstract}
In internal logistics the order-picking process is an important element of the material and information flow within a company - especially with regard to just-in-time deliveries and product liability. The selection of a suitable order-picking system is critical for the optimization of labour and time-consuming picking processes and thus helps to improve the performance while at the same time reducing the error rate.
\end{abstract}

Allerdings bedarf kaum eine Funktion in der gesamten Logistikkette ähnlicher Sorgfalt wie das Kommissionieren, da allgemeingültige Lösungsansätze oder branchenübergreifende „Patentlösungen“ kaum vorhanden sind.

Dort, wo sich technische Möglichkeiten zur Automatisierung ergeben und diese wirtschaftlich und ohne Einbuße an Flexibilität machbar sind, setzt sich diese auch für die Optimierung einzelner Prozesse durch. Von der rechnergesteuerten, automatischen Belieferung der Kommissionierplätze via Fördertechnik über die dynamische Bereitstellung von Nachschubeinheiten bis hin $\mathrm{zu}$ komplett vollautomatisierten Lösungen mit Kommissionierautomaten oder Kommissionierrobotern, die selbst den Pick-Vorgang übernehmen, wird alles am Markt angeboten.

Während bei einem hohem Durchsatz und relativ konstanter Auslastung ein hoher Automatisierungsgrad durchaus wirtschaftlich sein kann, profitieren Unternehmen, deren Kommissionierbedarf starken saisonalen Schwankungen unterliegt, eher von geringen Automatisierungsgraden in einem konventionellen Kommissioniersystem. Eine starke Automatisierung würde hier je nach Dimensionierung der Kommissionieranlage zu einer Unter- oder Überlastung des Systems und somit zu Leistungsschwankungen und einer im Mittel nur niedrigen Auslastung führen, die den Nachweis der Wirtschaftlichkeit deutlich erschwert.

Somit bestimmt die individuelle Analyse der gegebenen Kommissionieraufgaben unter Berücksichtigung aller relevanten Parameter (z. B. Produkt- und Sortimenteigenschaften, Auftragsstruktur, gewünschte Gestaltung des Informationsflusses, räumliche Gegebenheiten) die effiziente Organisation und adäquate Ausgestaltung des Kommissioniersystems und den damit verbundenen Automatisierungsgrad.

Deswegen setzt auch heute noch die Hälfte der Anwender auf manuelle Kommissionierlösungen. Hier steht der Mensch mit seinen hohen sensomotorischen Fähigkeiten, seiner Flexibilität und Lernfähigkeit sowie seinem Kostenvorteil gegenüber Kommissionierautomaten im Mittelpunkt. Dabei kann er in verschiedenen Teilprozessen durch die Technologie unterstützt werden. Ob bei der Bewegung der Güter zur Bereitstellung, bei der Fortbewegung des Kommissionierers selbst oder z. B. beim Transport der Kommissioniereinheit können verschiedene Fördermittel und Bediengeräte eingesetzt werden.

Als besonders relevant neben dem Materialfluss etabliert sich die Gestaltung des Informationsflusses, der die Aufbereitung der Kundenaufträge, die Weitergabe des Kommissionierauftrages und die Quittierung umfasst. Die Informationsweitergabe wird dabei grundlegend in papierbehaftet und beleglos unterschieden. Die beleglose Kommissionierung soll hauptsächlich zur Erhöhung der Kommissionierleistung, Reduzierung der Kommissionierfehler und Erhöhung der Systemflexibiliät beitragen. Dabei kann auf verschiedene Technologien zurückgegriffen werden (Pick-by-Scan, Pick-by-Voice, Pick-by-Light, Pick-by-Weight etc.). 
Dabei spielt die korrekte Identifikation der Güter eine wesentliche Rolle. Hier kommen bereits seit einigen Jahren Identtechnologien wie verschiedene 1D- und 2D-Barcodes zum Einsatz. Zunehmend wird auch der Einsatz von RFID-Technologie in der Kommissionierung diskutiert und vereinzelt in Pilotprojekten untersucht. Auto-ID-Technologien können hier in verschiedenen Teilprozessen zur Unterstützung der Mitarbeiter eingesetzt werden. Eine Identifikation bei der Entnahme und Abgabe der Kommissioniereinheiten ermöglicht die Bildung von Teilprozessen mit Qualitätsprüfung.

Die Realisierung dieser Qualitätsprüfung als selbststeuernden Regelkreis ermöglicht die dezentrale Überprüfung und die Entlastung des zentralen Steuerungssystems.

Besonders effizient lassen sich dezentrale Regelkreise durch den Einsatz von RFID-Technologie realisieren. So können entscheidungsrelevante Daten auf dem mit dem Gut verknüpften Smart Label gespeichert und an Entscheidungspunkten abgefragt und gegebenenfalls ergänzt oder geändert werden.

Weiterhin ermöglicht diese Technologie die Identifikation der in der Kommissioniereinheit enthaltenen Entnahmeeinheiten, da eine direkte Sichtverbindung nicht erforderlich ist. Dabei muss den physikalischen Grenzen der Technologie durch umfassende Untersuchungen und Abstimmung der Systeme auf die jeweiligen Sortimente Rechnung getragen werden.

Die Integration eines RFID-Systems in den Kommissionierprozess kann den Schwachstellen, wie Entnahme falscher Waren oder Mengen, niedrige Kommissionierleistungen aufgrund von Prüf-, Zähl- und Suchaktivitäten entgegenwirken und ebenfalls eine zeitnahe Bestandsaktualisierung ermöglichen. Auto-ID-Technolgien, insbesondere die RFID-Technologie, ermöglichen darüber hinaus die Verfolgung der Güter über die gesamte Supply Chain hinweg: von der Kommissionierung der Waren durch automatische Erkennung, Auszeichnung von Artikeln und Gebinden sowie die Erstellung von Kommissionierlisten und lückenloses Tracking and Tracing der Sendung vom Hersteller bis zum POS.

Diese Bündelung von Möglichkeiten und Vorteilen hat das Fachgebiet Logistik (FLog) der Universität Dortmund unter der Leitung von Prof. Dr. R. Jansen zum Anlass genommen, um die Integration der Technologie in den Kommissionierprozess anwendungsnah umzusetzen und wissenschaftlich zu untersuchen. Als Anwendungsszenario wurde ein manuelles 2-dimensional mechanisiertes Mann-zur-Ware Kommissioniersystem zugrunde gelegt. Das zentrale Element hierbei ist ein Kommissioniergerät, das mit einem RFID-System ausgestattet ist. Zum Einsatz kommt ein Vertikal-Kommissioniergerät WAV-50 der Firma Crown, welches über zwei Ladeplattformen und ein Terminal der Firma Solcon verfügt, das über WLAN mit dem Lagerverwaltungssystem verbunden ist. An beiden Ladeplattformen sind Antennen der Firmen Feig und Scemtec installiert, welche die Erfassung von Transpondern, die an Produkten, Verpackungen oder Ladungsträgern appliziert sind, ermöglichen. Dabei kann die obere Ladeplattform mit der Kabine des Kommissionierers vertikal verfahren und eine Greifhöhe von fünf Metern erreichen. Die Transponder der entnommenen Waren, die auf einer der beiden Ladeplattformen abgestellt sind, werden identifiziert und mit der im Terminal gespeicherten Pickliste abgeglichen. Handelt es sich um eine falsche Ware oder wurden zu viele Waren entnommen, erfolgt eine Warnmeldung. Die ausgelesenen Daten und die daraus abgeleiteten Informationen über die abgestellten Waren werden über das mit Hilfe von Lastaufnehmern der Firma RAVAS ermittelte Gewicht verifiziert. Der Teilprozess Entnahme verfügt nun über eine Qualitätsprüfung, die zudem über einen dezentralen Regelkreis auf dem Terminal realisiert wird.

Nachdem die Entnahme der Objekte akzeptiert und vom Kommissionierer quittiert wurde, erfolgt eine Aktualisierung des Lagerbestandes. Weiterhin wird der Kommissionierer beim Abzählen der Waren durch die Terminalsoftware unterstützt, indem ihm mit einem Counter die noch zu entnehmende Teilezahl sowie die nächste Entnahmestelle angezeigt werden. 


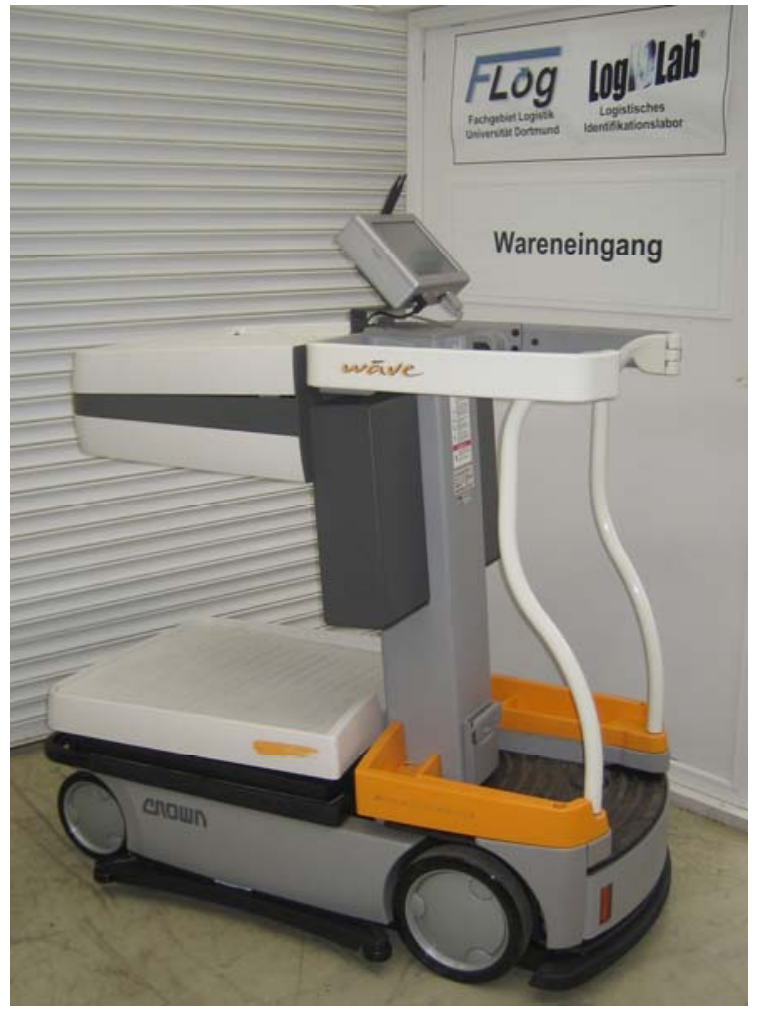

Abbildung 1: Detailansicht des RFID-gestützten Kommissioniergeräts

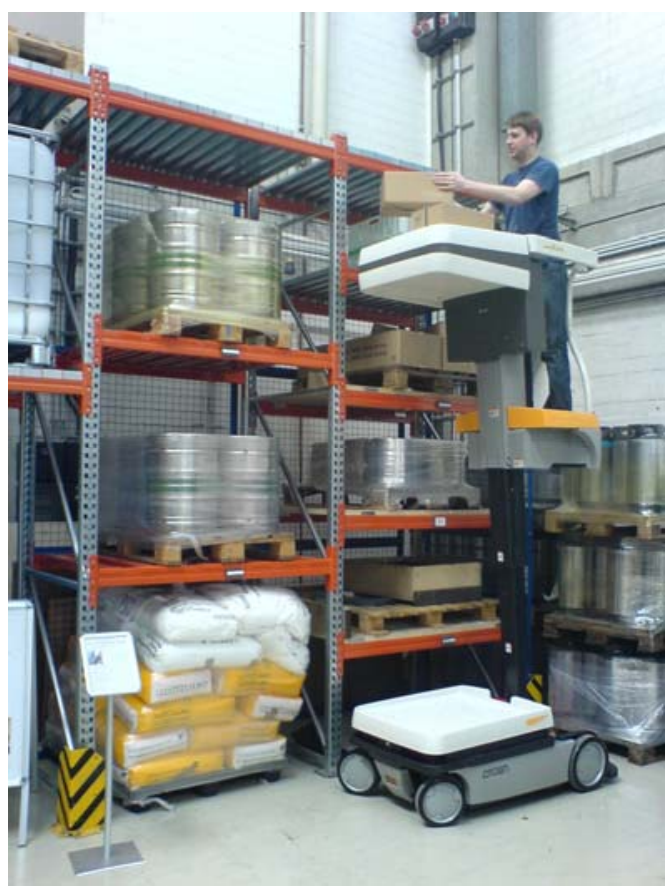

Abbildung 2: Kommissionierer im Einsatz 